\title{
¿QuiÉNES SON POBRES? ANÁLISIS de su IDENTIFICACIÓN EN AMÉRICA LATINA
}

\author{
Pablo Villatoro y María Emma Santos ${ }^{\mathrm{b}}$
}

Fecha de recepción: 22 de diciembre de 2018. Fecha de aceptación: 2 de mayo de 2019.

$$
\text { http://dx.doi.org/10.22201/iiec.20078951e.2019.199.68414 }
$$

Resumen. Este trabajo examina de forma empírica la solidez de identificación de los pobres mediante tres tipos de análisis usando datos de 2012 de cinco países de América Latina. Los resultados sugieren que el método monetario y el multidimensional nomonetario por separado son insuficientes para identificar la pobreza de manera comprehensiva, que parece conveniente el uso de umbrales de pobreza intermedios en el índice multidimensional y que la tipificación de grupos de pobreza hacia adentro de los países es sensible a la selección de dicho umbral. Lo anterior reafirma la necesidad de alcanzar consensos sobre la definición de pobreza en los países de la región.

Palabras clave: pobreza; método monetario; método multidimensional no-monetario; métodos de identificación; carencias sociales.

Clasificación JEL: I31; I32; P46.

\section{WHO IS POOR? AN ANALYSIS OF HOW PEOPLE LIVING IN POVERTY ARE IDENTIFIED IN LATIN AMERICA}

\begin{abstract}
This article examines empirically the soundness of how people living in poverty are identified, through three types of analysis and utilizing data from 2012 for five Latin American countries. The results demonstrate that the monetary method and the multidimensional non-monetary method alone are insufficient as a way to identify poverty in a comprehensive way. It is therefore appropriate to use intermediate poverty thresholds for the multidimensional index, and typification of poverty groups within these countries is sensitive to the selection of the threshold. These findings reaffirm the need to reach a consensus regarding the definition of poverty in the region's countries.
\end{abstract}

Key Words: poverty; monetary method; multidimensional non-monetary method; identification methods; social deprivation.

\footnotetext{
${ }^{a}$ Comisión Económica para América Latina y el Caribe (CEPAL), Chile; ${ }^{b}$ Instituto de Investigaciones Económicas y Sociales del Sur (IIEss), Departamento de Economía, uns-CONICET, Argentina, y opHI, University of Oxford, UK. Correos electrónicos: pablo.villatoro@cepal.org y msantos@uns.edu.ar, respectivamente. Los autores agradecen el financiamiento de ANPCyT-PICT 2015-2079 y de PGI-UNS 24/ZE30.
} 


\section{INTRODUCCIÓN}

El primer punto clave en la medición de la pobreza es la identificación de quiénes son pobres (Sen, 1976). Lo anterior presupone un concepto de pobreza en términos del espacio de medición, la unidad de análisis y el grado de lo absoluto $v s$. lo relativo en lo que representa ser pobre. A la identificación le sigue la agregación, la cual afecta la dirección en que se mueve el nivel estimado de pobreza ante modificaciones en los datos. Para efectos de análisis de este trabajo, el énfasis se pondrá en la etapa de identificación.

En América Latina, la medición predominante se ha hecho con el método del ingreso o línea de pobreza (LP), también llamado método indirecto por verificar el acceso al recurso, pero no la efectiva satisfacción de las necesidades, y -recientemente- método unidimensional, porque sólo considera el acceso al recurso monetario. Sin embargo, también hay en la región una tradición de medición con el método directo (Sen, 1981), por medio del enfoque de las Necesidades Básicas Insatisfechas (NBI), mediante el que se busca verificar si las personas satisfacen efectivamente un conjunto de necesidades básicas en diferentes dimensiones del bienestar. Durante los ańos ochenta, noventa y buena parte del 2000, muchos países han producido estadísticas oficiales por medio de ambos métodos.

En los últimos años, se ha evindeciado la necesidad de una evaluación más amplia de la pobreza, que no se limite al ámbito monetario. Entre los marcos conceptuales más influyentes se destacan el enfoque de derechos y de capacidades. El enfoque de derechos invoca la existencia de derechos humanos consagrados internacionalmente para avanzar en la garantía del cumplimiento de libertades fundamentales. Por ejemplo, la Declaración Universal de Derechos Humanos (1948) representó una bisagra fundamental que amplió los derechos hasta entonces reconocidos, incluyendo derechos económicos y sociales (Sen, 2009). Por su parte, el enfoque de capacidades (Sen, 2009) señala la importancia de evaluar el bienestar en el espacio de las capacidades o funcionamientos - fines del desarrollo- y no de los recursos -medios. Los funcionamientos son lo que una persona es efectivamente capaz de hacer en diferentes dimensiones de su vida (estatus nutricional, habilidades cognitivas, habilidades sociales, etcétera). Las capacidades son el conjunto de funcionamientos efectivamente disponibles para la persona.

Por otra parte, estudios participativos, como los de Narayan et al. (2000), reafirman que las personas pobres definen su condición de privación considerando las dimensiones que exceden a la de ingresos. Asimismo, los desarrollos 
teóricos en el ámbito de la medición multidimensional de la pobreza generan condiciones propicias para elaborar medidas de pobreza que cumplan con una serie de propiedades deseables (Atkinson, 2003; Bourguignon y Chrakravarty, 2003; Alkire y Foster, 2011, entre otros).

Así, las mediciones de pobreza por el método directo han cobrado nuevo impulso. Varios países de la región, dígase México (coneval, 2010), Colombia, Costa Rica, Chile, Ecuador, El Salvador, Honduras, Panamá y República Dominicana, elaboraron medidas multidimensionales de pobreza oficiales (Santos, 2019). También es frecuente la construcción de una tabla de contingencia que cruza la identificación de los pobres por el método monetario y el multidimensional no-monetario, produciendo una tipología que caracteriza a toda la población en función de la presencia o ausencia de privaciones monetarias y no-monetarias.

La coexistencia de dos índices oficiales de pobreza, uno monetario y otro no-monetario, enriquece la comprensión del problema, al tiempo que complejiza la respuesta a la pregunta central desde el punto de vista del diseño de políticas: ¿quién es pobre? Esto ocurre no sólo porque los dos índices de pobreza no necesariamente identificarán a la misma población como pobre, sino también, porque las decisiones sobre los criterios de identificación en ambos índices siempre pueden ser objeto de discusión.

En este trabajo se examina cuán sensible es la cantidad y la homogeneidad del grupo de personas identificadas como pobres a variaciones en los criterios de identificación, en un contexto caracterizado por la disponibilidad de un índice de pobreza por ingresos, y otro de pobreza por carencias críticas. Variar los criterios de identificación es equivalente a modificar las definiciones operativas de pobreza y, por ende, pone a prueba la necesidad y relevancia de llegar a consensos respecto de lo que significa ser pobre.

Existe una literatura importante respecto de la sensibilidad de las estimaciones de pobreza por ingresos a variaciones, tanto en la línea de pobreza (identificación), como en las medidas de agregación, lo cual ha dado origen al llamado enfoque de dominio estocástico, mismo que se considera toda la distribución, sea uni o multidimensional y que, trabajando con las funciones de distribución acumulada, intenta determinar si una distribución es preferida a la otra. El enfoque permite generar ordenamientos de pobreza, aunque incompletos, puesto que no siempre es posible determinar el dominio de una distribución por sobre otra (Foster y Sen, 1997).

Por otra parte, Székely et al. (2000) estudian la sensibilidad de las medidas de pobreza por ingresos a decisiones metodológicas tales como el uso de esca- 
las de adulto equivalente, la adopción de economías de escala en el consumo, el tratamiento de ingresos faltantes y cero, y el ajuste por subdeclaración de ingresos para el caso de 17 países de América Latina.

Este trabajo comparte la misma motivación que la bibliografía citada y busca aportar evidencia en un área de sensibilidad de las estimaciones de pobreza aún poco explorada: aquella que resulta de cruzar el método del ingreso con el del método directo o multidimensional no-monetario. Para ello, se tomó la identificación de pobreza monetaria como exógenamente dada y se analizaron variaciones en las estimaciones a partir de cambios en el umbral de pobreza multidimensional no-monetario.

El análisis se realizó con base en datos de cinco países de América Latina: Brasil, Colombia, México y El Salvador, para 2012, y Bolivia para 2011. El ejercicio podría haberse realizado con datos de otros años o países de la región, exponiendo discrepancias similares. Este conjunto de datos ofrece un nivel de comparabilidad bueno.

El trabajo es sólo una aproximación a la gran cantidad de alternativas y modificaciones que pueden explorarse. Aun así, es valioso en tanto ejemplifica la variabilidad de las magnitudes estimadas a las definiciones de pobreza y, por ende, representa un llamado a ofrecer la máxima transparencia metodológica posible, a avanzar en definiciones de pobreza consensuadas, y a la realización de pruebas de robustez y sensibilidad.

El documento se estructuró de la siguiente manera: en la sección dos se describe la identificación de la pobreza con el método de línea de pobreza, el multidimensional y el de tabla de contingencia. En la sección tres se presentan los resultados del análisis empírico. En la sección cuatro discuten alternativas para la determinación del umbral de pobreza en el método multidimensional y en la sección cinco se presentan las conclusiones.

\section{MÉTODOS DE IDENTIFICACIÓN DE LA POBREZA}

\section{El método del ingreso}

La medición de pobreza por línea de pobreza en América Latina consiste en estimar el costo de la canasta básica alimentaria (СвА) o "línea de indigencia" (LI), que satisface los requerimientos nutricionales promedio de la población, 
y el costo de la canasta básica total (Свт) o "línea de pobreza" (LP), que es la СвA ampliada por la inversa del coeficiente de Engel. ${ }^{1}$

¿Quiénes son los pobres de acuerdo con el método del ingreso? Pues aquellos cuyo ingreso sea inferior al de la línea de pobreza, es decir: si $y_{i}<z_{Y}$, donde $y_{i} \in \mathbb{R}_{+}$es el ingreso de la persona $i$, y $z_{Y}$ es la línea de pobreza. En términos de agregación, la medida con frecuencia utilizada es la tasa de la pobreza, definida como la cantidad de personas identificadas como pobres por LP, $q_{L P}$, como proporción del total poblacional $n$ :

$$
H_{L P}=q_{L P} / n
$$

La tasa de pobreza tiene la desventaja de que no registra incrementos de la pobreza cuando una persona pobre se empobrece más, así como tampoco cuando se produce una transferencia regresiva entre personas pobres (Sen, 1976). Sin embargo, para los efectos de este trabajo, esta medida es suficiente.

La determinación de la condición de pobreza por ingresos requiere una gran cantidad de decisiones como: la determinación de los requerimientos nutricionales, la selección de la población de referencia, la definición de los ítems de la СвА, el procedimiento para estimar el coeficiente de Engel, el uso del ingreso $v$ s. el consumo como indicadores de recursos y los procedimientos para corregir dichas variables (imputaciones por datos faltantes y ajustes por subdeclaración). Székely et al. (2000) muestran que la proporción de personas pobres en 17 países de América Latina va de 12.7 a 65.8\% del total de población considerada dependiendo de los procedimientos utilizados en algunos de los puntos mencionados.

\section{El método directo o multidimensional}

La forma tradicional de implementación del método directo en América Latina, es el enfoque de necesidades básicas (iniciado con Kast y Molina, 1975; INDEC, 1984). Sin embargo, recientemente se desarrollaron en la región nuevas medidas de pobreza por el método directo siguiendo la metodología de Alkire y Foster (AF) (2011), los cuales incluyen las medidas oficiales de varios países de la región, así como el índice de pobreza multidimensional para la región de América Latina propuesto en CEPAL (2014), Santos et al. (2015) y Santos y Villatoro (2018).

1 La inversa del coeficiente de Engel es el Gasto Total del Hogar dividido por el Gasto en Alimentos. Este coeficiente se calcula para un grupo poblacional de referencia, el cual puede ser definido de acuerdo con diferentes criterios. 
El método de AF comparte con el método de NBI un enfoque de conteo del número de privaciones para la identificación de la pobreza, por lo que se pueden presentar de manera conjunta. Suponiendo que la pobreza es evaluada en $d$ indicadores. Cada persona tiene un logro en cada indicador, el cual es comparado con un umbral de satisfacción en ese indicador. El primer paso para la identificación de quiénes son pobres requiere determinar si la persona exhibe privación en cada indicador considerado. En segundo término, la privación de cada persona es ponderada por el peso del indicador. A partir de lo anterior, se suman las privaciones ponderadas, obteniendo un puntaje de privación para cada persona llamado $c_{i}$.

¿Quiénes son los pobres en el método multidimensional? Los que experimentan una suma ponderada de privaciones igual o mayor a un umbral de privación, denotado con $k: c_{i} \geq k$. El umbral $k$ especifica la proporción de privaciones mínimas que una persona debe experimentar para ser considerada pobre. $k$ puede variar desde un criterio unión -requiere experimentar al menos una privación-, hasta un criterio intersección -requiere experimentar todas las privaciones consideradas. El $k$ puede definirse equivalente a proporción de privaciones que se experimentan.

En el método NBI se define a una persona como pobre (o con NBI) si habita en un hogar que exhibe al menos una NBI de una lista que por lo general incluye hacinamiento, vivienda inadecuada, ausencia de retrete, niños que no asisten a la escuela y un indicador de baja capacidad económica (alto índice de dependencia en el hogar y bajo nivel educativo del jefe de hogar). Como medida de agregación, el método NBI utiliza la proporción de personas con una o más NBI:

$$
H_{N B I}=q_{N B I} / n
$$

Cabe aclarar que los Institutos de Estadísticas y Censos de los países suelen reportar estadísticas desagregadas como la proporción de personas por cantidad y tipo de privaciones (véase INE, 2012).

El método NBI ha recibido críticas como: 1) la selección de los indicadores utilizados, subordinada a la disponibilidad de datos en las fuentes censales y a su correlación con la pobreza monetaria; 2) la equi-ponderación de los indicadores, lo cual implica cierto desbalance de ponderación dimensional (varios indicadores refieren a la calidad habitacional); 3) la utilización de un criterio de unión. Al utilizar la tasa de pobreza, el índice NBI ignora la profundidad (distancia al umbral de privación) y amplitud (cantidad de privaciones) de la pobreza (véase Feres y Mancero, 2001). 
La metodología de AF ofrece la posibilidad de superar algunas de estas limitaciones, ya que admite umbrales de pobreza $(k)$ intermedios, y permite que el índice sea sensible a la amplitud de la pobreza. La primera medida propuesta por AF es la tasa de recuento ajustada $M_{0}$. Esta medida combina dos subíndices: la proporción de personas multidimensionalmente pobres y la intensidad de su privación, la cual está dada por la proporción promedio de las privaciones (ponderadas) que experimentan quienes fueron identificados como pobres. Se puede expresar entonces la proporción de personas pobres de acuerdo con el umbral $k$ como $H_{k}=q_{k} / n$, donde, $q_{k}$ es el número de personas identificadas como pobres con el umbral $k$. La intensidad está dada por $A=\sum_{i=1}^{n} c_{i}(k) / q$, donde $c_{i}(k)$ es la suma de privaciones ponderadas de quienes fueron identificados como pobres. $M_{0}$ es el producto de estas dos medidas:

$$
M_{0}=H_{k} \times A
$$

La estructura de la medida $M_{0}$ se utiliza para el diseño de las medidas multidimensionales oficiales de pobreza en la mayoría de los países de la región y en el índice regional presentado en Santos et al. (2015) (IPM-LA de ahora en adelante).

El presente trabajo emplea una variante del IPM-LA que retiene 12 de los 13 indicadores, todos excepto el ingreso, puesto que precisamente el objetivo es evaluar las mediciones de pobreza no-monetaria en su cruce con las mediciones de pobreza monetaria. Este índice se llamará NBI-Ampliado. A su vez, se consideró un índice alternativo compuesto por 6 indicadores el cual se aproxima a la configuración tradicional del índice NBI. Este índice se llamará NBI. Por simplicidad y a diferencia del IPM-LA, se optó en ambos casos por equiponderar las privaciones (no las dimensiones). Los indicadores y ponderaciones de ambos índices se detallan en el cuadro 1. Estas dos medidas se refieren a índices de carencias sociales, puesto que ninguna de ellas contiene al ingreso como indicador.

Cabe señalar que el umbral de pobreza $k$ constituye un parámetro de gran importancia (equivalente a la línea de pobreza en la medición monetaria), que refleja en buena medida la conceptualización de pobreza subyacente. Por supuesto, esto atañe no exclusivamente al $k$ en sí mismo, sino en su configuración con la cantidad de indicadores y umbrales utilizados. 
Pablo Villatoro y María Emma Santos

Cuadro 1. Índices de carencias sociales utilizados

\begin{tabular}{|c|c|c|c|}
\hline \multirow[t]{2}{*}{ Dimensiones } & \multirow{2}{*}{$\begin{array}{l}\text { Indicadores } \\
\text { Hogares con o que: }\end{array}$} & \multicolumn{2}{|c|}{ Ponderación } \\
\hline & & Índice NBI-Ampliado & Índice NBI \\
\hline VIVIENDA & & 25 & 33.3 \\
\hline $\begin{array}{l}\text { Materiales de la } \\
\text { vivienda }^{a}\end{array}$ & $\begin{array}{l}\text { Viviendas con piso de tierra o con techo o muros con materiales } \\
\text { precarios (desechos, cartón, latas, caña, palma, paja, otros materiales). }\end{array}$ & 8.3 & 16.6 \\
\hline Hacinamiento & Tres o más personas por cuarto, en áreas rurales y urbanas. & 8.3 & 16.6 \\
\hline $\begin{array}{l}\text { Tenencia insegura } \\
\text { de la vivienda }\end{array}$ & $\begin{array}{l}\text { 1) habitan viviendas ocupadas ilegalmente, } 02 \text { ) residen en viviendas } \\
\text { cedidas o prestadas. }\end{array}$ & 8.3 & 0 \\
\hline
\end{tabular}

SERVICIOS BÁSICOS

$25 \quad 33.3$

Carencia de fuentes

Áreas urbanas:

$8.3 \quad 16.6$

de agua mejoradas Obtienen agua de alguna de las siguientes fuentes:

- red pública fuera del terreno;

- pozos no protegidos o sin bomba o motor;

- fuentes móviles (aljibe, carro tanque, aguatero, otros);

- agua embotellada, 0

- río, quebrada, lluvia y otros.

Áreas rurales:

Obtienen agua de alguna de las siguientes fuentes:

- pozos no protegidos o con bomba manual;

- fuentes móviles (alijibe, carro tanque, aguatero, otros);

- agua embotellada, 0

- río, quebrada, lluvia y otros.

Carencia de Áreas urbanas:

saneamiento

mejorado

- con evacuación no conectada a red de alcantarillado o fosa séptica, 0

- con baño compartido, 0

- que no disponen de servicio higiénico.

Áreas rurales:

- que no disponen de servicio higiénico;

- con baño compartido, 0

- con evacuación sin tratamiento a la superficie, río o mar.

Carencias de No tienen servicio eléctrico o usan leña, carbón o desechos como

energía combustible para cocinar.

EDUCACIÓN 


\begin{tabular}{|c|c|c|c|}
\hline \multirow[t]{2}{*}{ Dimensiones } & \multirow{2}{*}{$\begin{array}{l}\text { Indicadores } \\
\text { Hogares con o que: }\end{array}$} & \multicolumn{2}{|c|}{ Ponderación } \\
\hline & & Índice NBI-Ampliado & Índice NBI \\
\hline $\begin{array}{l}\text { Logro educativo } \\
\text { insuficiente }\end{array}$ & $\begin{array}{l}\text { Ninguna persona de } 20 \text { años o más alcanzó un nivel educativo mínimo, } \\
\text { entendiéndose por ello lo siguiente: } \\
\text { - personas de entre } 20 \text { y } 59 \text { años: no cuentan con el primer ciclo de la } \\
\text { educación secundaria completo, y } \\
\text { - personas de } 60 \text { años o más: no cuentan con educación primaria completa. }\end{array}$ & 8.3 & 16.6 \\
\hline Rezago escolar & $\begin{array}{l}\text { Al menos un niño o adolescente (entre } 6 \text { y } 17 \text { años) está rezagado en el } \\
\text { sistema educativo en más de dos años de acuerdo con su edad. }\end{array}$ & 8.3 & 0 \\
\hline ESTÁNDAR DE VIDA & & 8.3 & \\
\hline $\begin{array}{l}\text { Carencia de bienes } \\
\text { duraderos }\end{array}$ & $\begin{array}{l}\text { No cuentan con ninguno de los siguientes bienes: 1) vehículo, } \\
\text { 2) refrigerador y 3) lavadora. }\end{array}$ & 8.3 & 0 \\
\hline \multicolumn{2}{|c|}{ EMPLEO Y PROTECCIÓN SOCIAL } & 16.6 & 0 \\
\hline Desocupación & $\begin{array}{l}\text { Al menos una persona de entre } 15 \text { y } 65 \text { años está en alguna de las } \\
\text { siguientes situaciones: } \\
\text { - desempleada; } \\
\text { - empleada sin remuneración, } 0 \\
\text { - es un trabajador desalentado. }\end{array}$ & 8.3 & 0 \\
\hline $\begin{array}{l}\text { Precariedad de } \\
\text { la seguridad } \\
\text { sociall }\end{array}$ & $\begin{array}{l}\text { Presentan al menos una de las siguientes situaciones: } \\
\text { - ninguna persona cuenta con algún tipo de seguro de salud contributivo; } \\
\text { - ninguna persona está afiliada a un sistema de previsión social contributivo; } \\
\text { - ninguna persona tiene ingresos por pensiones o jubilaciones }\end{array}$ & 8.3 & 0 \\
\hline
\end{tabular}

Notas: ${ }^{a}$ No se dispuso de información sobre piso para Brasil (2012); ${ }^{b}$ No se considera privación la habitación en viviendas recibidas en usufructo; ' No se dispuso de información sobre seguro de salud para Brasil (2012). Se consideró solamente el indicador de seguridad social.

Fuente: elaboración propia, en base a Santos et al. (2015).

\section{El método integrado, bidimensional o de tabla de contingencia}

A mediados de la década de los ochenta comienzan a utilizarse la tabla de contingencia entre la medición de pobreza por NBI y la de línea de pobreza (LP), llamado por Beccaria y Minujin (1985) y Kaztman (1989) el "método integrado" (también conocido como enfoque bidimensional), para evaluar si las incidencias de pobreza obtenidas por los distintos métodos eran similares (Boltvinik, 2013). La constatación de diferencias importantes entre las estimaciones generadas por ambos métodos llevó a la construcción de una tipología ex post. Se entendió entonces que el método LP no captaba necesidades que no requieren de gasto y que el método NBI no era apto para captar necesidades que pueden satisfacerse con el ingreso. 
Cuadro 2: Tabla de contingencia de la pobreza monetaria y no-monetaria

\begin{tabular}{llll}
\hline \multicolumn{4}{c}{ NBI o carencias no-monetarias } \\
\hline Monetaria (LP) & Pobre & No pobre & Total \\
\hline Pobre & $n_{11}$ & $n_{10}$ & $n_{1+}$ \\
No pobre & $n_{01}$ & $n_{00}$ & $n_{0+}$ \\
Total & $n_{+1}$ & $n_{+0}$ & $n$ \\
\hline
\end{tabular}

Fuente: con base en Alkire et al. (2015), cap. 2.

Las categorías consideradas en la primera versión del enfoque bidimensional pueden describirse de la siguiente forma (Feres y Mancero, 2001): a) pobres crónicos $\left(n_{11}\right)$ : hogares "consistentemente" pobres (Ringen, 1987), que formarían el "núcleo duro" de la pobreza, $b$ ) pobres inerciales $\left(n_{01}\right)$ : integrado por sujetos que habrían dejado recientemente la pobreza por ingresos, pero que mantienen carencias no- monetarias y, $c$ ) pobres recientes $\left(n_{10}\right)$ : quienes han visto caer sus ingresos bajo la línea de pobreza, como consecuencia de algún impacto económico, pero que no tienen carencias sociales. Así el método bidimensional, al menos en la interpretación de Kaztman (1989) y CEPAL/ DGEC (1988), no siempre tiene asociado un criterio estricto de identificación de la pobreza, sino más bien ofrece una tipificación que puede ser útil al diseño de política.

La reciente difusión de nuevos desarrollos en el campo de la medición multidimensional de la pobreza ha renovado el interés en el uso de tablas de contingencia, siendo su principal expresión oficial la medición multidimensional vigente en México. También deben mencionarse la propuesta de la Comisión para la Medición de la Pobreza en Chile (2014) y un trabajo del MDs Brasil (2015).

Cabe notar que en las aplicaciones recientes en México, Chile y Brasil se efectuaron algunos cambios respecto de la interpretación original del método de la tabla de contingencia. Por ejemplo, en México (coneval, 2010) se definen como pobres multidimensionales a quienes son pobres por ingreso y experimentan al menos una carencia social (los que en la interpretación tradicional eran pobres crónicos). Quienes son pobres por ingresos, pero no por carencias, son llamados vulnerables por ingresos, en tanto que, quienes pertenecen a la categoría inversa son llamados vulnerables por carencias sociales. A su vez, tanto en México como en Chile, se generó un subgrupo de extremadamente pobres, definidos por tener ingresos bajo la línea de indigencia y ser pobre por carencias sociales. 


\section{Fuentes de datos}

Las estimaciones se realizaron para cinco países de la región -Brasil, Colombia, México y El Salvador- en 2012, excepto Bolivia en el que los datos corresponden a 2011 debido a su disponibilidad al momento de realizar los cómputos. Los países fueron seleccionados atendiendo a la completitud de la información y a la variabilidad de la incidencia de la pobreza. En el cuadro 3 se presentan las fuentes de datos y el porcentaje de personas identificadas como pobres para cada umbral $k$ en los dos índices de carencias sociales.

Cuadro 3. Fuentes de datos e incidencias de la pobreza por carencias sociales. Índices de 12 y de 6 privaciones (porcentajes de población)

\begin{tabular}{|c|c|c|c|c|c|c|c|c|}
\hline \multirow[t]{4}{*}{ Países } & \multirow[t]{4}{*}{ Fuente y año } & \multicolumn{5}{|c|}{ Índice 12 privaciones (\%) } & \multicolumn{2}{|c|}{ dice 6 privaciones (\%) } \\
\hline & & \multicolumn{4}{|c|}{ Cantidad y proporción de carencias } & \multicolumn{3}{|c|}{ Cantidad y proporción de carencias } \\
\hline & & $K=1$ & $K=2$ & $K=3$ & $K=4$ & $K=1$ & $K=2$ & $K=3$ \\
\hline & & (8.3) & $(16.7)$ & (25) & (33.3) & $(16.7)$ & (33.3) & (50) \\
\hline Brasil & $\begin{array}{l}\text { Pesquisa Nacional } \\
\text { por Amostra de } \\
\text { Domicilios } 2012\end{array}$ & 60 & 31 & 15 & 6 & 44.5 & 13.8 & 3.3 \\
\hline Colombia & $\begin{array}{l}\text { Gran Encuesta } \\
\text { Integrada de Hogares } \\
2012\end{array}$ & 76 & 53 & 34 & 22 & 48.5 & 21.9 & 9.6 \\
\hline México & $\begin{array}{l}\text { Encuesta Nacional } \\
\text { de Ingresos y Gastos } \\
\text { de los Hogares } 2012\end{array}$ & 80 & 59 & 42 & 28 & 62 & 36 & 17 \\
\hline El Salvador & $\begin{array}{l}\text { Encuesta de Hogares } \\
\text { de Propósitos } \\
\text { Múltiples } 2012\end{array}$ & 85 & 69 & 55 & 42 & 68 & 45 & 27.5 \\
\hline Bolivia & $\begin{array}{l}\text { Encuesta Continua } \\
\text { de Hogares } 2011\end{array}$ & 93 & 81 & 66 & 51 & 81 & 55 & 35 \\
\hline
\end{tabular}

Nota: $k=$ umbral de pobreza por carencias sociales; $k=1$ implica que basta que las personas tengan alguna carencia para ser clasificadas como pobres; $k=2$ implica que se requieren como mínimo 2 carencias para que las personas sean consideradas pobres.

Fuente: elaboración propia. Las bases de datos provienen del Banco de Datos de Encuestas de Hogares (BADEHOG) de la CEPAL. 


\section{RESULTADOS DEL ANÁLISIS}

\section{Concordancia entre la identificación monetaria y no-monetaria de la pobreza}

Las medidas monetarias y no-monetarias son distintas y, por tanto, es de esperar que exista cierta discrepancia. Sin embargo, también es deseable alguna concordancia, puesto que cada medición capta distintas expresiones de un mismo fenómeno. En otros términos, si dos medidas de pobreza son concordantes en su totalidad, cualquiera de los dos métodos podrá ser usado para medir pobreza, sin necesidad de combinarlos. A su vez, si las dos medidas de pobreza producen clasificaciones discrepantes en su totalidad, es que están captando fenómenos distintos.

La forma más simple de analizar la concordancia de dos métodos que miden el mismo fenómeno, es calcular el número total de observaciones coincidentes como porcentaje del total de observaciones. Dada la notación presentada en el cuadro 2, la concordancia se define como:

$$
C=\frac{n_{11}+n_{00}}{n}
$$

En el cuadro 4 se presentan los resultados del análisis simple de concordancia considerando distintos umbrales de carencias sociales, desde una carencia (criterio unión) hasta todas (criterio intersección).

Los datos que se exponen en el cuadro 4 muestran que los porcentajes de concordancia entre ambos métodos son menores en los niveles de $k$ más bajos, luego aumentan alcanzando su máximo para un $k$ de entre 3 y 5 carencias, y vuelven a bajar para los valores más exigentes de $k$. La excepción es Bolivia, donde recién en el tramo de 6 y 7 carencias se produce la mayor concordancia entre ambos métodos.

El comportamiento del nivel de concordancia entre ambos métodos con respecto al umbral de pobreza $k$ del índice multidimensional es intuitivo. Cuando se utiliza un criterio unión en el índice de carencias, es muy probable que alguien experimente una privación no-monetaria sin necesariamente ser pobre por ingresos: el $n_{01}$ tiende a ser alto y, por ende, el nivel de concordancia tiende a ser bajo. A medida que el umbral de pobreza $k$ aumenta, esta posibilidad disminuye: se hace menos probable que alguien experimente varias privaciones simultáneas y no sea pobre por ingresos. Es decir, el $n_{01}$ tiende a disminuir, y la concordancia comienza a aumentar. Sin embargo, a medida que se van alcanzando valores $k$ más y más altos, el $n_{10}$ tiende a aumentar (se 
Cuadro 4. Concordancias entre las medidas por LP y por carencias sociales a distintos valores de $k$ (porcentajes del total poblacional)

\begin{tabular}{lrrrrr}
\hline $\begin{array}{l}\text { Umbral de carencias } \\
\text { sociales }(k)\end{array}$ & $\begin{array}{r}\text { Brasil } \\
2012\end{array}$ & $\begin{array}{r}\text { Colombia } \\
2012\end{array}$ & $\begin{array}{r}\text { México } \\
2012\end{array}$ & $\begin{array}{r}\text { El Salvador } \\
2012\end{array}$ & $\begin{array}{r}\text { Bolivia } \\
2011\end{array}$ \\
\hline 1 & 54.1 & 54.9 & 53.1 & 57.0 & 42.3 \\
2 & 75.3 & 70.4 & 64.6 & 64.6 & 51.6 \\
3 & 82.3 & 75.2 & 69.3 & 68.6 & 60.7 \\
4 & 83.4 & 75.3 & 70.9 & 70.0 & 67.7 \\
5 & 82.6 & 73.5 & 69.7 & 68.8 & 71.6 \\
6 & 82.0 & 71.6 & 68.3 & 65.7 & 72.8 \\
7 & 81.6 & 70.0 & 65.8 & 62.8 & 72.8 \\
8 & 81.5 & 68.7 & 64.0 & 59.2 & 70.8 \\
9 & 81.5 & 67.9 & 63.3 & 56.8 & 68.0 \\
10 & 81.5 & 67.4 & 63.0 & 55.3 & 65.3 \\
11 & 81.5 & 67.2 & 62.9 & 54.6 & 63.8 \\
12 & 81.5 & 67.1 & 62.8 & 54.5 & 63.7 \\
\hline
\end{tabular}

Nota: para valores de $k$ altos ( 9 y más) la concordancia se estabiliza porque las personas en pobreza monetaria comienzan a ser captadas inequívocamente por la pobreza no-monetaria, y se estabiliza el porcentaje de personas pobres por carencias sociales.

Fuente: elaboración propia.

hace más probable que alguien sea pobre por ingresos, sin que necesariamente exhiba un alto número de privaciones no-monetarias). Así, nuevamente, la concordancia disminuye.

Un problema del indicador de concordancia basado en el porcentaje de acuerdo entre dos métodos es que una parte de la concordancia observada puede ser fruto del azar. Una medida que intenta corregir ese aspecto es el coeficiente Kappa (Cohen, 1960), diseñado para medir la correlación intraclase y definido como:

$$
k=\frac{P o-P e}{(1-P e)}
$$

donde $P o$ es la concordancia observada $(P o=C$ ) y $P e$ es la concordancia esperada debido al azar. En términos de la notación incluida en el cuadro 2, la concordancia esperada se calcula como la suma de los productos de las probabilidades de que ambos métodos clasifiquen a una persona como pobre y la probabilidad de que ambos métodos clasifiquen a una persona como nopobre: 


$$
P e=\left(\frac{n_{1+}}{n}\right) *\left(\frac{n_{+1}}{n}\right)+\left(\frac{n_{0+}}{n}\right) *\left(\frac{n_{+0}}{n}\right)
$$

Cuando hay concordancia perfecta, Kappa es igual a 1. Si la concordancia observada es igual a la esperada por azar, Kappa es 0, y los valores negativos (muy inusuales) indican un acuerdo inferior al azar. Valores del coeficiente Kappa inferiores a 0.2 son usualmente interpretados como insignificantes; valores entre 0.2 y 0.4 , como concordancia baja; valores entre 0.4 y 0.6 como concordancia moderada y valores mayores a 0.6 como concordancia alta (Viera y Garrett, 2005).

En la gráfica 1 se aprecia que -al igual que con el coeficiente C- la concordancia entre ambos métodos medida por el coeficiente Kappa es mayor en los umbrales $k$ intermedios de carencias sociales (principalmente 3 y 4 privaciones), y es más baja para los umbrales de carencias más extremos. También puede observarse que los niveles de concordancia con el coeficiente Kappa son menores que con la medida $\mathrm{C}$, sugiriendo que una parte de la concordancia de $\mathrm{C}$ es en realidad azarosa.

El hecho de que aún para niveles intermedios del umbral de pobreza por carencias sociales, la concordancia sea moderada, implica que ambas medidas están captando parcialmente el mismo fenómeno, pero también que ninguna de ellas es suficiente por sí sola para captar adecuadamente la pobreza.

Gráfica 1. Concordancia entre las medidas por LP y por carencias sociales, cambiando los umbrales multidimensionales de carencias sociales

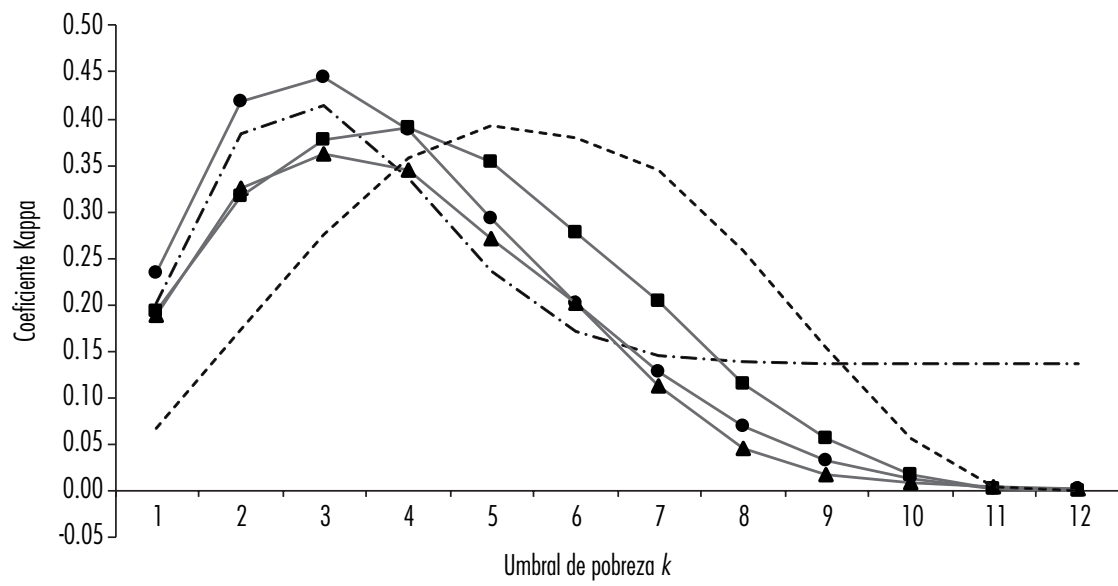

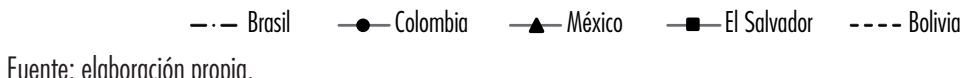


Cabe destacar que se ha tomado la identificación por línea de pobreza como exógenamente dada y válida. Sin embargo, es sabido que la determinación de dicho umbral también está sujeto a una gran cantidad de decisiones metodológicas que afectan el grado de concordancia con las medidas multidimensionales no-monetarias.

\section{Sensibilidad de la identificación a variaciones en el umbral de pobreza multidimensional}

Esta sección evalúa el efecto de variaciones en el umbral y en la cantidad de indicadores de carencias en el índice multidimensional sobre la identificación de las categorías generadas por el método bidimensional. En total se examina la robustez del método de tabla de contingencia usando 7 especificaciones alternativas: el índice versión NBI (6 indicadores) con $k$ de 1 hasta 3 y el índice versión NBI-Ampliado (12 indicadores) con $k$ desde 1 hasta 4 . Dado el número de indicadores, a mayor $k$, menor probabilidad de ser identificado como pobre. A su vez, dado el $k$, es menos probable ser identificado como pobre con el índice versión NBI que con el NBI-Ampliado puesto que tiene menor cantidad de indicadores. Cabe señalar que un $k=1, k=2, k=3, k=4, k=5$ y $k=6$ en el Índice NBI es, respectivamente, comparable a un $k=2, k=4, k=6, k=8, k=10 \mathrm{y}$ $k=12$ en el NBI-Ampliado, en tanto demandan privaciones del 16.7, 33, 50, 66.7, 83.3 y $100 \%$, respectivamente.

En la gráfica 2 (compuesta de cuatro gráficos) se presenta el ordenamiento de los países para cada uno de los cuatro grupos definidos por la tabla de contingencia presentada en el cuadro 1, para las diferentes especificaciones del índice multidimensional. Se aprecia que los ordenamientos de los países según la incidencia de cada grupo tienden a mantenerse al variar el umbral $(k)$ y la cantidad de indicadores en el índice multidimensional. Lo que es más evidente para los grupos de "pobres por ambos métodos" y "no pobres por ambos métodos". La robustez de las estimaciones de los grupos "inconsistentes" (pobres por LP y no pobres por carencias y viceversa) es algo menor: sólo se puede afirmar sin ambigüedad que Bolivia es el país donde el grupo de pobres por carencias y no pobres por LP tiene una mayor incidencia y el grupo de pobres por LP y no pobres por carencias tiene una menor incidencia, independientemente del valor de $k$ y de la cantidad de indicadores.

En la gráfica 3 se presentan las mismas incidencias, pero en lugar de comparar la incidencia de cada grupo en los diferentes países al variar el índice multidimensional, se compara la incidencia de los diferentes grupos en cada país. 


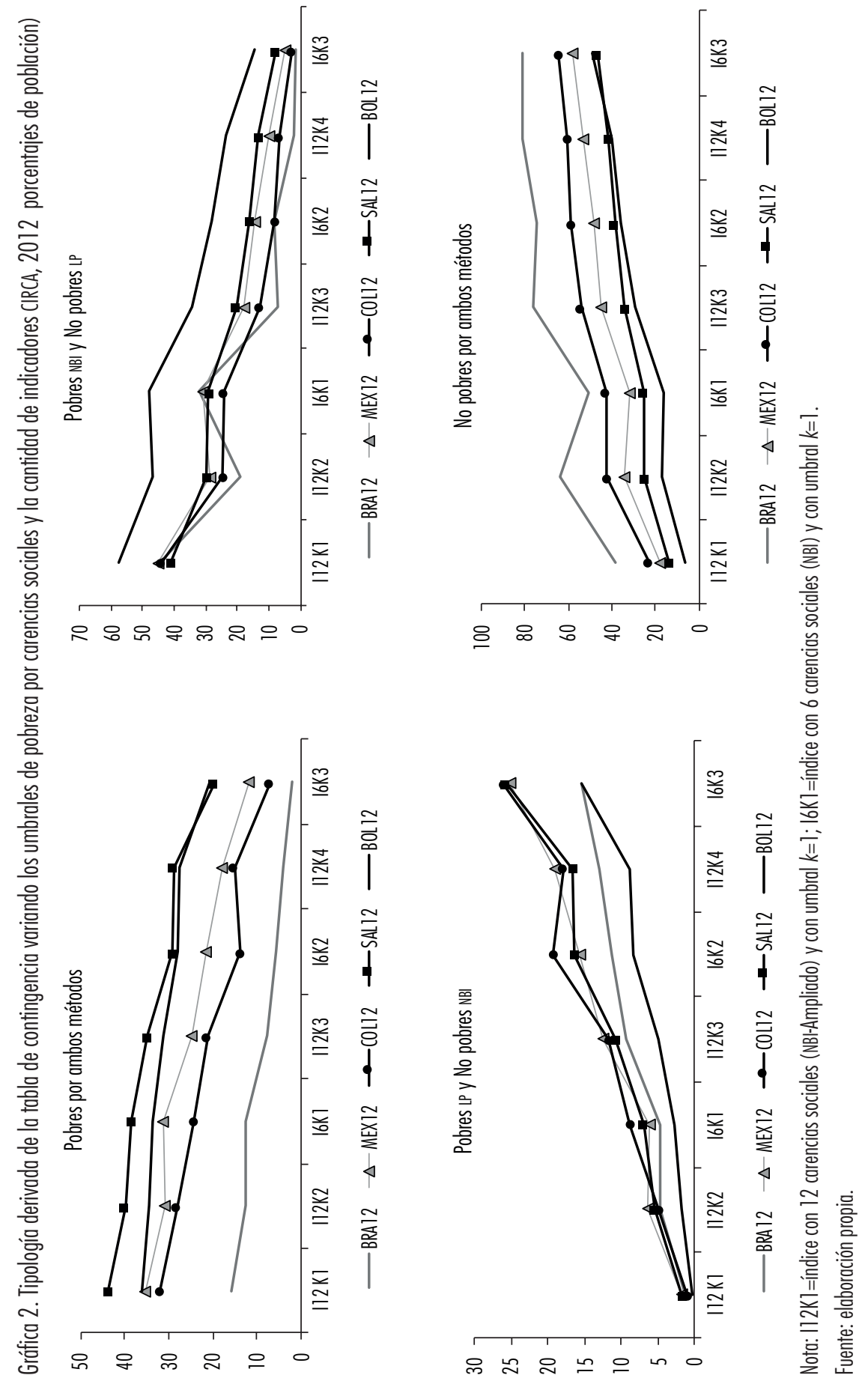


Gráfica 3. Incidencia de los distintos tipos de pobreza dentro de los países, variando umbrales y cantidad de indicadores (porcentajes de población)

Brasil 2012

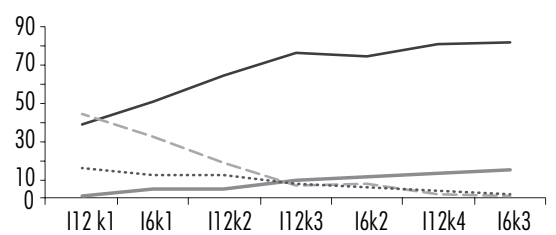

— No pobres por ambos métodos — Pobres LP y no pobres carencias

--- Pobres carencias y no pobres LP ...... Pobres por ambos métodos
México 2012

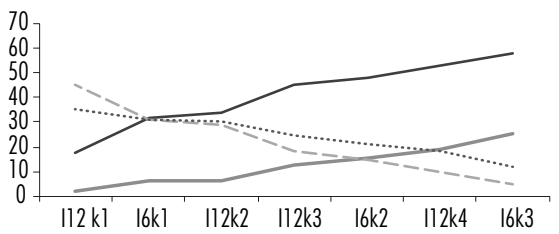

— No pobres por ambos métodos — Pobres LP y no pobres carencias

--- Pobres carencias y no pobres LP ...... Pobres por ambos métodos

\section{Colombia 2012}

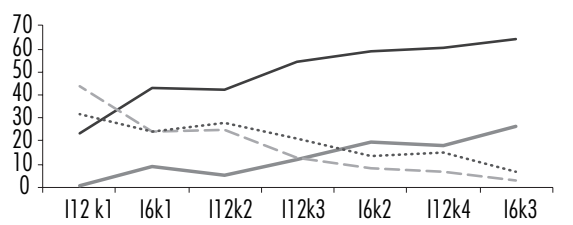

— No pobres por ambos métodos — Pobres LP y no pobres carencias

--- Pobres carencias y no pobres LP ...... Pobres por ambos métodos
El Salvador 2012

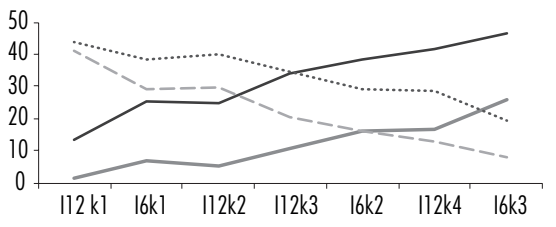

— No pobres por ambos métodos — Pobres LPy no pobres carencias

--- Pobres carencias y no pobres LP $\quad$...... Pobres por ambos métodos

Bolivia 2011

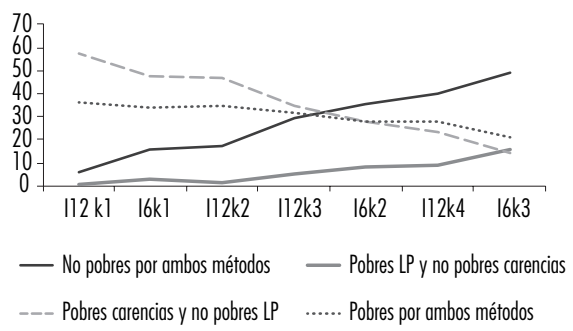

Nota: II 2K1=índice con 12 carencias sociales $y$ con umbral k=1; 16K1=índice con 6 carencias sociales y con umbral $k=1$. Fuente: elaboración propia. 
Como es de esperar, al variar el umbral de pobreza en el índice multidimensional y la cantidad de indicadores considerados, el ordenamiento de los distintos grupos identificados en cada país cambia de forma significativa.

La tendencia de la incidencia de cada grupo tiene el mismo comportamiento en cada país y está dado por definición. $\mathrm{Al}$ incrementarse el valor de $k$ y al reducirse el número de indicadores del índice, la proporción de personas pobres por ambos métodos disminuye, con un incremento en la de no-pobres por ambos métodos como contrapartida. A su vez, la proporción de personas que son pobres por NBI, pero no por LP, también disminuye, y aumenta como contraparte la proporción de personas pobres por LP, pero no por NBI. En este marco, si el umbral de pobreza por carencias sociales es muy poco exigente (por ejemplo, criterio unión), y además se usa una mayor cantidad de indicadores de carencias, la probabilidad de que se identifique a alguien como no pobre por carencias sociales se reducirá sustancialmente.

Esto explica lo que sucede con la especificación $\mathrm{I} 12 / \mathrm{K}=1$, con la cual el grupo de pobres por ingresos y no pobres por carencias casi desaparece. Así, esta especificación tiene una menor aptitud para captar los efectos de las crisis económicas. En efecto, es probable que muchas de las personas que caen en la pobreza monetaria experimenten pocas carencias no-monetarias durante un tiempo. Asimismo, un $k$ muy bajo y una mayor cantidad de indicadores de carencias no-monetarias pueden llevar a sobreestimar el grupo de no pobres por ingreso y pobres por NBI.

\section{Homogeneidad intra-grupal}

Al definir los grupos de personas con privaciones por el método integrado se espera no sólo que cada grupo sea distinto del otro, sino que cada grupo exhiba cierto grado de homogeneidad interna en aquellos atributos críticos para afirmar que los sujetos clasificados en un grupo pertenecen sin ambigüedad a dicho grupo y no a otro.

En la gráfica 4 se presenta la incidencia de las carencias sociales en el grupo "pobres por carencias sociales y no pobres por ingreso", y "pobres por ambos métodos", utilizando la especificación de $k=1$ y el índice de 12 privaciones. Los porcentajes presentados suman 100\% dentro de cada grupo. Los datos muestran bastante variabilidad en la incidencia de carencias sociales dentro de cada grupo. Por ejemplo, quienes son no pobres por ingreso y sólo tienen una carencia social conforman más de la mitad del grupo en Brasil, $44 \%$ en Colombia, 36\% en México, 28\% en El Salvador y 18.7\% en Bolivia. 
Gráfica 4. Incidencia de carencias sociales en grupos seleccionados, $k=1^{0}$ (porcentajes de población dentro de cada grupo) ${ }^{b}$

Brasil 2012

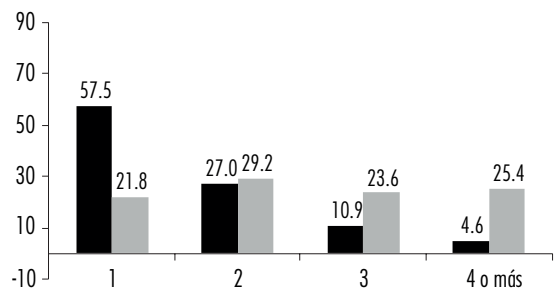

- Pobre carencias y No pobre ingresos $\quad$ Pobre por ambos métodos

México 2012

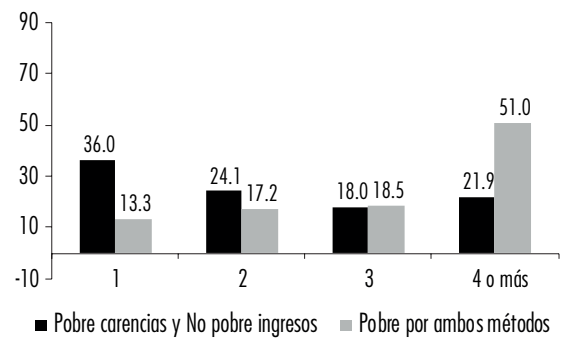

El Salvador 2012

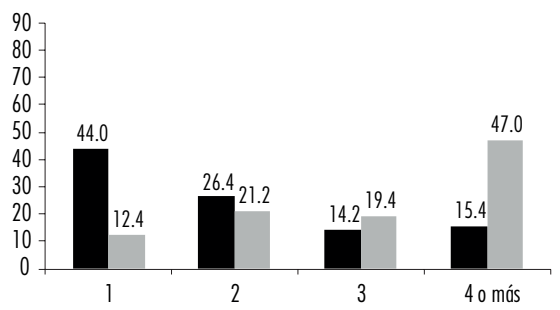

- Pobre carencias y No pobre ingresos $\approx$ Pobre por ambos métodos

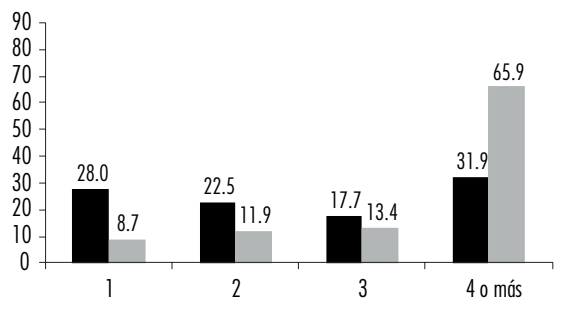

- Pobre carencias y No pobre ingresos $\square$ Pobre por ambos métodos

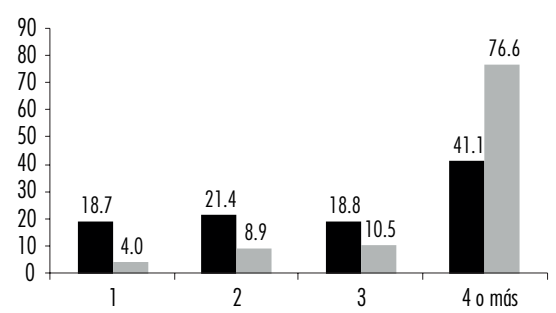

- Pobre carencias y No pobre ingresos $\backsim$ Pobre por ambos métodos

Notas: ${ }^{a} \mathrm{~K}=1$ significa al menos 1 carencia en el índice de 12 carencias sociales; ${ }^{b}$ Porcentaje de población dentro de cada grupo afectada por 1, 2, 3, 4 o más carencias sociales. Los porcentajes suman 100\% dentro del grupo "Pobre por carencias y No pobreza por ingresos" y dentro del grupo "Pobre por ambos métodos".

Fuente: elaboración propia. 
Por su parte, quienes presentan 3 privaciones sociales o más (sumando los grupos de 3 privaciones y 4 o más) alcanzan $15 \%$ en Brasil, $29.6 \%$ en Colombia, 39.9\% en México, 49.6\% en El Salvador y 59.9\% en Bolivia. Así, dentro de cada grupo hay una gradación de intensidad de la pobreza dada por la cantidad de privaciones que se experimentan, lo que sugiere que el grupo de "pobres inerciales" en el esquema de Kaztman engloba a personas en diversidad de condiciones.

También se observa heterogeneidad entre quienes son pobres por ambos métodos: los que tienen 4 privaciones o más representan 25\% en Brasil, $47 \%$ en Colombia, 51\% en México, 65.9\% en El Salvador y 76.6\% en Bolivia, lo que evidencia la gradación en términos de intensidad de la pobreza. Es de esperar que dentro de este grupo también exista una gradación en cuanto a la profundidad de la privación por ingresos.

Interpretado de otra manera, las cifras cuestionan no solamente la adecuación de la tipología del método integrado, sino también problematizan ambas formas de medición. Por una parte, el que un sujeto no sea pobre por LP, pero experimente 1 de 12 carencias lleva a problematizar la precisión de la identificación por el lado de las carencias cuando se utiliza un criterio de unión $(k=1)$; por otra parte, el que un sujeto esté privado en 3 o más indicadores no monetarios y al mismo tiempo no sea pobre por LP lleva a cuestionar la identificación por LP.

En la gráfica 5 se presentan las incidencias de las privaciones sociales en los cuatro grupos derivados de la tabla de contingencia clásica, con base en el índice de 12 privaciones, pero con un $k=3$. Los porcentajes de población con diferente número de carencias suman $100 \%$ dentro de cada grupo. Aunque aquí se utiliza un umbral de pobreza $k$ más exigente, la heterogeneidad interna de los grupos persiste. Por ejemplo, al considerar el grupo de "no pobres por ambos métodos" fluctúa entre el 51\% (Brasil) y el 22\% (Bolivia), y dentro del mismo grupo, quienes tienen 2 privaciones no-monetarias presentan incidencias entre el $42 \%$ (Bolivia) y el 16\% (Brasil). Cabe preguntarse entonces, cómo deberían ser clasificados estos últimos sujetos si es que sus ingresos estuvieran muy cerca del umbral de pobreza.

El grupo de "pobres por ingresos y no pobres por carencias" exhibe heterogeneidad: la incidencia de quienes tienen 2 privaciones está alrededor o sobre el 50\%. Por su parte, la alta incidencia de personas con 4 carencias o más en este grupo lleva a preguntarse si es razonable que este subgrupo no sea considerado como "consistentemente pobre" (por no presentar privación monetaria), siendo que quienes tienen 3 carencias y están privados en ingresos son clasificados como "consistentemente pobres". Por último, en el grupo 
Gráfica 5. Incidencia de carencias sociales en grupos seleccionados, $k=3^{a}$

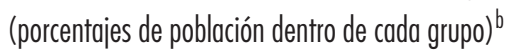

Brasil 2012

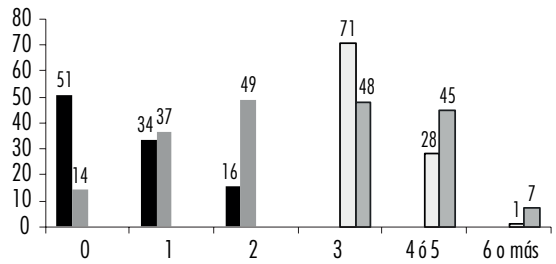

- No pobre por ambos métodos - Pobre ingresos y No pobre carencias $\square$ Pobre carencias y No pobre ingresos aPobre por ambos métodos
El Salvador 2012

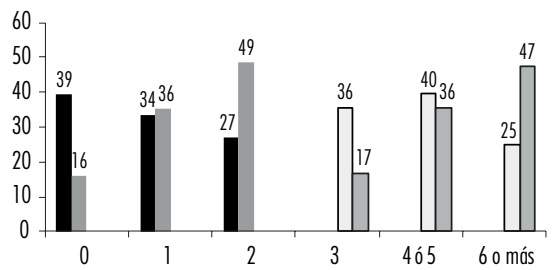

- No pobre por ambos métodos $\quad$ Pobre ingresos y No pobre carencias

$\square$ Pobre carencias y No pobre ingresos $\square$ Pobre por ambos métodos

\section{Bolivia 2011}

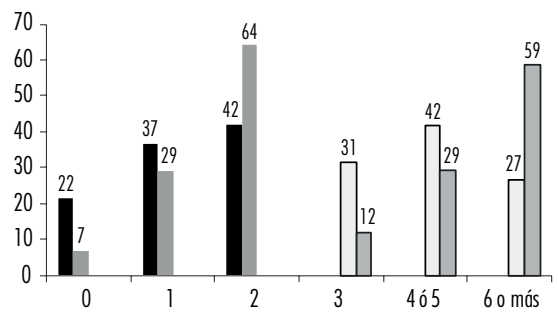

- No pobre por ambos métodos $\quad$ Pobre ingresos y No pobre carencias

$\square$ Pobre carencias y No pobre ingresos 口Pobre por ambos métodos

Notas: ${ }^{a} \mathrm{~K}=3$ implica un umbral multidimensional de pobreza por carencias sociales de 3 carencias; ${ }^{b}$ Porcentaje de población dentro de cada grupo afectada por 0, 1, 2, 3, 4, 5 ó 6 o más carencias sociales. Los porcentajes suman 100\% dentro de cada grupo (No pobre por ambos métodos; Pobre por ingresos y No pobre por carencias; Pobre por carencias y No pobre por ingresos; Pobre por ambos métodos).

Fuente: elaboración propia. 
"pobre por ambos métodos", se podría dudar sobre la adecuación de clasificar a quienes tienen 6 privaciones o más en el mismo grupo donde están quienes son afectados solamente por 3 privaciones.

Aun reconociendo el problema de la heterogeneidad intragrupal, también es preciso reconocer que existe una tensión entre la precisión de la identificación y la operacionalidad del criterio. El total de posibles configuraciones de privaciones aumenta de forma exponencial al incrementarse el número de indicadores considerados. La agregación en un grupo con algún grado -aunque imperfecto- de homogeneidad, resulta necesaria para hacer operativa la medición y las acciones de política pública.

\section{REFLEXIONES EN TORNO AL UMBRAL DE POBREZA $K$}

El ejercicio realizado para 2012 ejemplifica la sensibilidad de las estimaciones de pobreza ante variaciones en los criterios de identificación, sensibilidad que también se observaría si se utilizaran otros años de análisis. Este análisis promueve la discusión sobre algunos puntos fundamentales del criterio de identificación de la pobreza.

En primer lugar, los resultados que se muestran en la sección concordancia entre la identificación monetaria y no-monetaria de la pobreza sugieren que la concordancia entre la identificación de la pobreza por medio del índice de carencias y la identificación de la pobreza por medio del método de LP es -a lo sumo- moderada, lo cual es coincidente con evidencia encontrada en otros trabajos tanto sobre América Latina como sobre países de otras regiones. Esto sugiere que ambas medidas proveen información parcial y deberían ser combinadas de alguna manera.

Una alternativa de combinación es definir a las personas pobres como aquellas que son consistentemente pobres (pobres por el criterio monetario y el no-monetario). Ello implica asociar la noción de pobreza sólo a una de las tres posibles situaciones de carencia que se puede identificar mediante el método de tabla de contingencia. Este es -en la práctica- el camino que ha seguido México en su medición oficial, ya que para ser identificado como pobre se requiere necesariamente experimentar privación de ingresos además de al menos una privación en carencias sociales. Otra posibilidad es utilizar como criterio de identificación la unión de los conjuntos identificados como pobres por ambas medidas (Boltvinik, 2013). No obstante, esto podría dar lugar a incidencias de pobreza muy altas, haciéndolo poco operativo como información para el diseño de políticas selectivas. La tercera alternativa es incluir 
la dimensión monetaria en un índice multidimensional, sin establecer como requisito la pobreza monetaria para la identificación de la pobreza.

En cualquier caso, el índice multidimensional requiere la fijación del umbral de pobreza $k$. La evidencia presentada en en este trabajo indica la importancia de la determinación del $k$ sobre todo para la cuantificación y tipificación de la pobreza hacia dentro de cada país. La magnitud relativa de los grupos con las distintas combinaciones de privaciones monetarias y no-monetarias dentro de cada país cambia sustancialmente al variar el umbral $k$, lo que presenta un desafío para cualquier forma de combinación de ambos índices.

¿De qué maneras puede definirse el umbral de pobreza multidimensional $k$ ? Una opción es la definición estrictamente normativa, desde una perspectiva de derechos, según la cual la presencia de una sola carencia basta para que exista una situación de pobreza. Esta alternativa no está exenta de discrecionalidad en la selección de los derechos y umbrales a observar. Pobreza no es igual a la violación de cualquier derecho humano (оHCHR, 2003), y esta falta de identidad conceptual obligará a tomar decisiones respecto de los derechos que serán incluidos y los que no. A su vez los problemas de disponibilidad de datos podrían llevar a excluir derechos fundamentales (por ejemplo, el derecho a la alimentación). También se deberán tomar decisiones respecto de los umbrales que garantizarían los derechos. En la práctica, el contenido del derecho internacional puede interpretarse como norma (aplicación literal) o como principio, en cuyo caso se toman en cuenta las capacidades de los Estados para garantizar el derecho, atendiendo a la idea de progresividad de aplicación.

El criterio de unión fundado en la perspectiva de derechos ha sido el caso de la medida multidimensional oficial de México: cada una de las 6 carencias que constituyen la parte no-monetaria del índice multidimensional se entiende como un derecho constitucional. Sin embargo, para ser identificado como multidimensionalmente pobre, se requiere además experimentar pobreza monetaria. Así, puede argumentarse que implícitamente este criterio relativiza la aplicación del enfoque de derechos a la privación en el espacio de la disponibilidad de ingresos. A su vez, presenta el problema de que la población afectada por varias carencias no-monetarias al mismo tiempo, pero no pobre por ingresos, no será identificada como pobre multidimensional.

Por otra parte, la utilización de un criterio de unión puede estar sujeta a mayores errores de medición que la utilización de un umbral más alto. En efecto, es más probable que una persona aparezca como privada en un indicador por un error de medición, o bien, que dicha privación sea voluntaria y no forzada, a que lo mismo ocurra con dos o más privaciones. La evidencia de este trabajo sugiere que el criterio unión en el índice de carencias sociales 
presenta problemas desde el punto de vista empírico: 1) tiene valores de concordancia muy bajos con la pobreza monetaria, 2) exhibe baja capacidad de identificación de situaciones de empobrecimiento reciente, 3) presenta altos niveles de heterogeneidad intragrupal.

Una alternativa distinta para la fijación del $k$ es determinarlo de manera empírica, evaluando la distribución de incidencias de pobreza que se obtiene para sus diferentes valores. En estos casos, por lo general, se hace uso de algún criterio de validación externo, ya sea una medición independiente del mismo fenómeno, tal como podría ser la tasa de pobreza monetaria, o bien una definición consensuada por un conjunto de actores en torno al nivel de pobreza esperado. Dicha medida de validación debe gozar de respaldo y bajo error de medición. Los IPM oficiales de la región desarrollados hasta ahora utilizan un valor de $k$ intermedio (con variedad de valores), elegido por lo general de manera positiva o empírica, aunque utilizando criterios normativos en la selección de los demás parámetros que interactúan con el $k$ (Santos, 2019), y realizando análisis de robustez en los ordenamientos de pobreza ante variaciones en el $k$.

\section{CONCLUSIONES}

La evidencia de este trabajo favorece el uso de un método de identificación de la pobreza que combine la dimensión monetaria con dimensiones no-monetarias y, a su vez, la utilización de un umbral de pobreza multidimensional intermedio el cual debería fundarse en un criterio consensuado con la máxima participación posible de los actores implicados, y debería ser sometido a escrutinio por medio de un criterio de validación externo.

La implicancia de la evidencia presentada es que la estimación del grupo de personas en situación de pobreza es eminentemente contingente a la conceptualización de pobreza que se tenga, lo cual no debe desalentar de ninguna manera la medición de pobreza, en tanto es un ejercicio fundamental para el avance en el desarrollo humano. Lo que debe entenderse es la importancia de una definición consensuada respecto de quiénes deben ser considerados pobres:

No es extraño que la discusión del problema de la pobreza esté plagada de diferencias de criterio[...] Es concebible -aunque no necesario- que pese a estas diferencias originales del esquema valorativo se pueda llegar a un acuerdo amplio sobre la norma de pobreza aplicable en una sociedad [...] Pero aun cuando exista 
un considerable grado de consenso social sobre los niveles mínimos de bienestar, no es tarea fácil para el especialista en ciencias sociales identificar y explicitar ese consenso (Sen, 1978, citado en Altimir, 1979, pp. 7-8).

En esta línea sería importante desarrollar lo siguiente: 1) herramientas prácticas que permitan la generación de consensos sobre concepto y umbrales de pobreza, tales como espacios de diálogo y concertación, con representación de los distintos actores sociales involucrados en el problema de la pobreza, y 2) modos más sistemáticos y objetivos para "describir las prescripciones prevalecientes" (Altimir, 1979, p. 8), para lo cual podría avanzarse en la implementación de encuestas de necesidades socialmente percibidas (Mack y Lansley, 1985, estudios cualitativos, estudios participativos y rondas de consulta de manera regular. Es entorno a esa definición consensuada y explicita de lo que representa ser pobre en la región de América Latina hoy, que debe continuar avanzando, para que las mediciones sean fieles a tales nociones y, por ende, las acciones de política sean efectivas.

\section{BIBLIOGRAFÍA}

Alkire, S. y Foster, J. (2011), "Counting and multidimensional poverty measurement”, Journal of Public Economics, vol. 95, núm. 7-8. Elsevier, agosto. Alkire, S., Foster, J. E., Seth, S., Santos, M. E., Roche, J. M. y Ballon, P. (2015), Multidimensional poverty measurement and analysis, Oxford, University Press.

Altimir, O. (1979), "La dimensión de la pobreza en América Latina”, Cuadernos de la CEPAL, núm. 27, Santiago de Chile.

Atkinson, A. B. (2003), "Multidimensional deprivation: contrasting social welfare and counting approaches", Journal of Economic Inequality, vol. 1, núm. 1, Springer, abril.

Banco de Datos de Encuestas de Hogares (BADehog), Comisión Económica para América Latina y el Caribe.

Beccaria, L. y Minujin, A. (1985), "Métodos alternativos para medir la evolución del tamaño de la pobreza", Documentos de Trabajo 6, Buenos Aires, Instituto Nacional de Estadística y Censos.

Boltvinik, J. (2013), "Medición multidimensional de pobreza. América Latina de precursora a rezagada”, Revista Sociedad \& Equidad, núm. 5, Universidad de Chile, enero. 
Bourguignon, F. y Chakravarty, S. R. (2003), "The measurement of multidimensional poverty”, Journal of Economic Inequality, vol. 1, núm. 1, Springer, abril.

Cohen, J. A. (1960), “Coefficient of agreement for nominal scales”, Educational and Psychological Measurement, vol. 20, núm. 1, sAGE Journals, abril.

Comisión Económica para América Latina y el Caribe (CEPAL) (2014), Panorama Social de América Latina 2014, Santiago de Chile, CEPAL.

Comisión Económica para América Latina y el Caribe (CEPAL)/Dirección General de Estadística y Censos del Uruguay (DGEC) (1988), "La heterogeneidad de la pobreza: una aproximación bidimensional". Recuperado de <http://repositorio.cepal.org/bitstream/handle/11362/28222/LCmvdR12rev1_es.pdf?sequence $=1>$

Comisión para la Medición de la Pobreza (2014), Informe Final Comisión para la Medición de la Pobreza, Santiago de Chile, enero. Recuperado de <http://www.cl.undp.org/content/chile/es/home/library/poverty/infor mes_de_comisiones/informe-final--comision-para-la-medicion-de-lapobreza.html>

Consejo Nacional de Evaluación de la Política de Desarrollo Social (Coneval) (2010), Metodología para la medición multidimensional de la pobreza en México, México, coneval. Recuperado de <https://www.coneval.org.mx/ rw/resource/Metodologia_Medicion_Multidimensional.pdf>

Feres, J. C. y Mancero, X. (2001), "El método de las necesidades básicas insatisfechas (NBI) y sus aplicaciones en América Latina”, Serie Estudios Estadísticos y Prospectivos 7, Santiago de Chile, Publicación de las Naciones Unidas, LC/L.1491-P.

Foster, J. E. y Sen, A. (1997), On economic inequality after a quarter century, Oxford, Oxford University Press.

Instituto Nacional de Estadística (INE) (2012), Necesidades Básicas Insatisfechas. Cuadros Estadísticos, Bolivia. Recuperado de <https://www.ine.gob. bo/index.php/podreza-desarrollo/introduccion-3>

Instituto Nacional de Estadísticas y Censos (INDEC) (1984), La pobreza en la Argentina. Indicadores de Necesidades Básicas Insatisfechas a partir de los datos del Censo Nacional de Población y Vivienda 1980, Argentina, Presidencia de la Nación, Secretaría de Planificación.

Kast, M. y Molina, S. (1975), Mapa de la extrema pobreza, Chile, Odeplan, Escuela de Economía Pontificia Universidad Católica.

Kaztman, R. (1989), "La heterogeneidad de la pobreza. El caso de Montevideo", Revista de la CEPAL, núm. 37, CEPAL, abril. 
Mack, J. y Lansley, S. (1985), Poor Britain, London, Allen and Unwin.

Ministério de Desenvolvimento Social e Combate à Fome (MDS) (2015), "Pobreza Multidimensional: uma análise a partir do índice proposto pela Comissão Econômica para América Latina e Caribe (CEPAL)", Estudo Técnico, núm. 02/2015. Recuperado de <https://fpabramo.org.br/acervoso cial/estante/pobreza-multidimensional-uma-analise-partir-indice-proposto-pela-comissao-economica-para-america-latina-e-caribe-cepal/>

Narayan, D., Chambers, R., Shah, M. K. y Petesch, P. (2000), Voices of the poor: crying out for change, Washington, DC, World Bank.

OHCHR (2003), Human rights and poverty reduction. A conceptual framework. Recuperado de <http://www.ohchr.org/Documents/Publications/PovertyReductionen.pdf>

Ringen, S. (1987), The possibility of politics, Oxford, Oxford University Press.

Santos, M. E. (2019), "Desafíos en el diseño de Índices de Pobreza Multidimensional Nacionales", Serie de Estudios Estadísticos de la CEPAL, núm. 100, CEPAL.

Santos, M. E. y Villatoro, P. (2018), "A multidimensional poverty index for Latin America", Review of Income and Wealth, vol. 64, núm. 1, Wiley, marzo.

Santos, M. E., Villatoro, P., Mancero, X. y Gerstenfeld, P. (2015), "A Multidimensional poverty index for Latin America", OPHI Working Paper 79, Oxford, Oxford University.

Sen, A. (1976), "Poverty: an ordinal approach to measurement", Econometrica, vol. 44, núm. 2, Wiley, marzo.

(1978), "Three notes on the concept of poverty", Income distribution and employment programme, WEP 2-23/WP 65, Ginebra, Oficina Internacional del Trabajo.

(1981), Poverty and famines. An essay on entitlement and deprivation, Oxford, Oxford University Press.

(2009), The idea of justice, New York, Penguin Books.

Székely, M., Lustig, N., Cumpa, M. y Mejía, J. A. (2000), “Do we know how much poverty there is?", Working Paper, núm. 437, Washington D.C., Banco Interamericano de Desarrollo, Departamento de Investigación.

Viera, A. J. y Garrett, J. M. (2005), "Understanding interobserver agreement: the Kappa statistic", Family Medicine, vol. 37, núm. 5, FTMI, mayo. 
